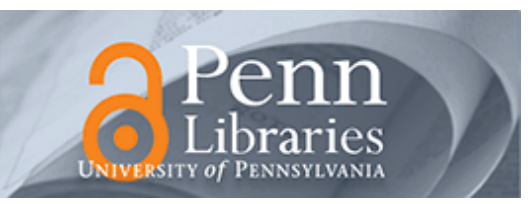

University of Pennsylvania

ScholarlyCommons

May 2003

\title{
Satisfying the Perceived Need for Free-Play in Pedagogically Oriented Interactive Dramas
}

\author{
Barry G. Silverman \\ University of Pennsylvania, basil@seas.upenn.edu \\ Michael Johns \\ University of Pennsylvania \\ Ransom Weaver \\ University of Pennsylvania
}

Follow this and additional works at: https://repository.upenn.edu/ese_papers

\section{Recommended Citation}

Barry G. Silverman, Michael Johns, and Ransom Weaver, "Satisfying the Perceived Need for Free-Play in Pedagogically Oriented Interactive Dramas", . May 2003.

Copyright 2003 IEEE. Reprinted from Proceedings of the 16th IEEE International Conference on Computer Animation and Social Agents 2003 (CASA 2003), pages 161-167.

Publisher URL: http://ieeexplore.ieee.org/xpl/tocresult.jsp?isNumber=27000\&page=1

This material is posted here with permission of the IEEE. Such permission of the IEEE does not in any way imply IEEE endorsement of any of the University of Pennsylvania's products or services. Internal or personal use of this material is permitted. However, permission to reprint/republish this material for advertising or promotional purposes or for creating new collective works for resale or redistribution must be obtained from the IEEE by writing to pubs-permissions@ieee.org. By choosing to view this document, you agree to all provisions of the copyright laws protecting it.

This paper is posted at ScholarlyCommons. https://repository.upenn.edu/ese_papers/41

For more information, please contact repository@pobox.upenn.edu. 


\title{
Satisfying the Perceived Need for Free-Play in Pedagogically Oriented Interactive Dramas
}

\author{
Abstract \\ This research explores ways of harnessing people's passion for entertainment in order to stimulate \\ players to attain the meta-learning skills they need for lifelong learning and to reach the highest level of \\ learning, that of learning by teaching. More specifically to keep up with today's pace of training and the \\ goal for it to be just-intime, we are interested in creating a game generator to empower grass roots \\ experts to share their stories in such a way that others learn the lessons by engagement and empathy \\ with the storyworld characters. Doing so requires us to address the fundamental issue that caused the \\ entertainment and educational gaming communities to split: that it is not easy to create something fun \\ while constrained by rigid pedagogical goals. We examine a case study of the attempts of one cross- \\ disciplinary team to overcome aesthetic silos, and propose a critical systems methodology and generator \\ that harness the core mechanics that make gaming fun, while providing an accessible means for \\ educators to create content to deliver the educational payload.
}

\section{Keywords}

videogame generator, role playing games, story telling, training, stealth learning, systems approach

\section{Comments}

Copyright 2003 IEEE. Reprinted from Proceedings of the 16th IEEE International Conference on Computer Animation and Social Agents 2003 (CASA 2003), pages 161-167.

Publisher URL: http://ieeexplore.ieee.org/xpl/tocresult.jsp?isNumber=27000\&page=1

This material is posted here with permission of the IEEE. Such permission of the IEEE does not in any way imply IEEE endorsement of any of the University of Pennsylvania's products or services. Internal or personal use of this material is permitted. However, permission to reprint/republish this material for advertising or promotional purposes or for creating new collective works for resale or redistribution must be obtained from the IEEE by writing to pubs-permissions@ieee.org. By choosing to view this document, you agree to all provisions of the copyright laws protecting it. 


\title{
Satisfying the Perceived Need for Free-Play in Pedagogically Oriented Interactive Dramas
}

\author{
Barry G. Silverman, Michael Johns, Ransom Weaver \\ Ackoff Center for Advancement of Systems Approaches (ACASA) \\ University of Pennsylvania, Philadelphia, PA 19104-6315 \\ barryg@seas.upenn.edu
}

\begin{abstract}
This research explores ways of harnessing people's passion for entertainment in order to stimulate players to attain the meta-learning skills they need for lifelong learning and to reach the highest level of learning, that of learning by teaching. More specifically to keep up with today's pace of training and the goal for it to be just-intime, we are interested in creating a game generator to empower grass roots experts to share their stories in such a way that others learn the lessons by engagement and empathy with the storyworld characters. Doing so requires us to address the fundamental issue that caused the entertainment and educational gaming communities to split: that it $\dot{\mathrm{s}}$ not easy to create something fun while constrained by rigid pedagogical goals. We examine a case study of the attempts of one cross-disciplinary team to overcome aesthetic silos, and propose a critical systems methodology and generator that harness the core mechanics that make gaming fun, while providing an accessible means for educators to create content to deliver the educational payload.
\end{abstract}

Keywords: videogame generator, role playing games, story telling, training, stealth learning, systems approach

\section{Introduction and Goals}

A major purpose of this research is to explore alternative ways for a game generator to help authors introduce entertainment and free play into role playing games and interactive dramas that are training interventions. This goal arises since learner oriented game designs are one of the most difficult areas in developing videogames. First off, although training requires players to progress through stories (pedagogically valuable scenarios), at its heart game play is not about interactive fiction though there are those who buy interactive fiction games. Interactive drama is all about storytelling from the author, while game-play is much more about story creation by the player - and these competing aesthetics need to be resolved if pedagogical games are to achieve their potential in general.

Many game designers try to confront their players with a tradeoff decision, a dilemma. Thus you can't win a Pac-man level unless you risk your lives and collect all the food/energizers that the ghosts more or less protect.
Likewise in Space Invaders you score on offense, though you live longer by taking evasive tactics and going on defense. Making the tradeoff decisions (reluctantly answering the call), venturing forth to collect rewards (questing), and reaching the ultimate objective (conquering the enemy) - this sounds an awful lot like the "hero's journey" [1]. In fact, it is a hero's journey, one where the player is the hero. Without ever writing a single story, game designers are brilliant at inserting mechanics (dilemmas, actions, rewards) that allow players to create innumerable new stories. Because these stories are so different, yet exist about a common experience, the players readily share them with one another, compare them, brag about them, etc. And the players are encouraged to replay the game to live through new stories that they can further brag about.

More than any other mechanic of game-play, narrative in game raises the idea of destroying the central aesthetic - that players create their own stories and that is what keeps them coming back. Other game-play mechanics more or less have a story inside them, in fact countless stories inside them. Further, many of these mechanics have built in skill training functions at the same time that they permit unconstrained play and inquiry. For example, a racing and chasing mechanic includes lessons about how to chase down bad guys and cut them off from escape. Likewise a combat game has built in weapon firing and target damage models, plus skill challenges such as room clearing, among others. If one invests in the realism of these mechanics, they provide useful training and transferable skills [3].

It's also a fact that students learn the most and retain it the longest when they must teach a topic to others. One always learns a subject better if one is confronted with being the teacher rather than the student. So a microworld could be quite a powerful training device if it affords teaching opportunities, or even better if it thrusts the player into roles where other characters will be vulnerable and dependent on the player to teach for a successful conclusion to be reached.

Why should the player care to become a teacher? What can drive them to reach this level of learning? People reflect this kind of passion for videogames and at the movies. When game mechanics work and when characters are likable, players (viewers) achieve enormous empathy for the characters and are willing to go 
to great lengths to save them and to help them work out their problems (e.g., as in 'God' games such as The SIMS, virtual Petz, and Tamagotchi), and to go on quests on their behalf or assist them in shifting their behaviors to more successful models such as in role playing games. In these milieus, players reveal willingness to learn skills that will help the dysfunctional characters to cope.

\section{Creating Stories With Free Play}

As already mentioned, we are seeking to set up a generator that can expose constructs and parameters of a storyworld so that new interventions may be more readily authored that promote free play and entertainment within a narrative structure. To support this research, we are attempting to produce a cast of animated puppets and sets (introduced in what follows) in a way that they can be reused for many stories. This is the idea of a composable and reusable storyworld, including digital sets, cast members, and Campbellian archetypes that can be adapted, and extended for further sequels not even yet anticipated. Our ideas for reusable casts and archetypes follow from work such as $[1,2,14]$ as well as how they are used in franchise games, comics, and serials. We include characters of different ages, genders, and backgrounds/ethnicities, and in the roles of hero, sidekick, allies, opponents, tricksters, lovers, and so on.

It is worth pointing out that we made a conscious decision to base this cast and sets around 2-D, medium quality cartoon animations since research has shown that subjects with health behavior change issues often allocate little cognitive processing to health messages, and feel greater confidence about being able to process and conquer message sets introduced in cartoon formats [6].

In addition, we decided to use finite state machines as the basis for our dialog model, with nodes containing noninteractive sections of speech and gesture, and edges representing the various options available to the user after the node plays out. Within nodes, we opted for a tree structure with individual behaviors (speak, move, or gesture) as elements. Direct children of tree elements are executed in parallel immediately after an element finishes. This creates the turn-taking system, while still allowing for movement and gesture to occur in multiple characters simultaneously. The entire system is currently imple mented atop Director and Flash.

\subsection{Narrative Intelligence}

The field that some refer to as 'narrative intelligence' has recently produced a number of rich ideas for incorporating narrative into game worlds without totally sacrificing gaming's central aesthetic. In this research, we synthesize, adapt, and extend several of these ideas as this section will note. None of the research to date has directly addressed the topic of learning by teaching, so this places us in a new realm that drives our inquiry. Also, very little if any of the narrative intelligence research to date has addressed how to assist storyworld authors, so several original contributions are needed here as well to truly realize our research goals. We are creating the Authoring Edutainment Stories for Online Players (AESOP) generator as part of this research and are seeking to have it assist with authoring constructs as portrayed in Figure 1 and as further described in what follows.

From the player's perspective, when they encounter a storyworld such as in Figure 1a, they do not wish to be placed on 'rails' -- a storyline forcing the player down a narrow path that is author specified. The best narrative solutions found in the game field to date tend to approach this concern by interspersing free play/inquiry and player story-creation with player-selected choice points for advancing the story. At these choice points, the player approaches characters or other devices that reveal more of the author's story and that advance them to the next scene of the drama. In this manner, a drama eventually unfolds. Some successful examples of this blending of story and game are Grand Theft Auto and Deus Ex, among others.

Similar to this is the approach being taken in the Army's Mission Rehearsal Environment [16, 19], however, unlike the popular titles this approach is concerned with imparting doctrinally correct training objectives. MRE's approach requires authors to (1) deconstruct the story into the smallest parts (scene nodes) where autonomous character and player freeplay can be permitted, and (2) to identify graph transitions that are gate conditions for triggering scenes and/or for allowing scenes to be omitted without loss of training value. This approach permits the player to explore a node repeatedly, getting better with each try and through exit node feedback. However, this approach is for doctrinally correct training that requires repetitions for improvement.

In the current research we are interested in systems thinking, in mental model transfer, and in behavior shifting as mentioned earlier. This relaxes the need to repeat the identical scene, and affords the opportunity for scenes to hold surprising plot reversals if you play them differently. In storytelling theory, the listeners, or participants, are viewed as containing significant understanding and know-how already, and the story is but a fuse to ignite the recipients into synthesizing a new conceptualization for themselves. This is also consistent with persuasion theory, such as in the Persuasion Likelihood Model [13] which states that rational arguments are unlikely to persuade. Rather it is the peripheral cues that are modeled which convince the audience. Thus the many movie scenes of actors smoking during or after a significant activity have far more power than all the public health media campaigns laying out the rational arguments about adverse health effects. 
Figure 1 - Sample Insertion Points for Narrative Intelligence to Minimize Perceptions of Limiting Free Play

\section{(a) Plot is Path Chosen Through Storyworld}

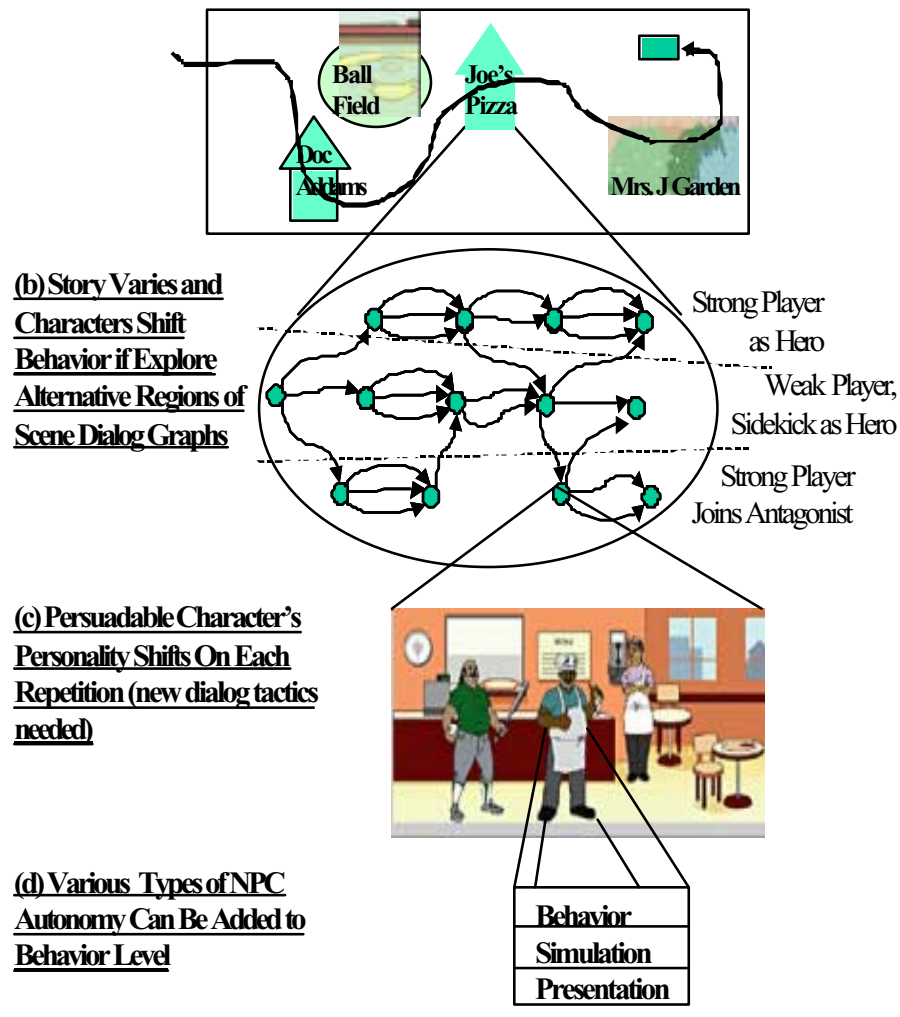

Figure $1 \mathrm{~b}$ zooms us in on a plot or dialog graph for a sample scene or quest of the storyworld. Here the nodes and edges are as described for the FSM in the prior section. Depending on the player's personal goals, entertainment objectives, style of play, and confidence, among other factors, they may decide to pursue very different avenues through a scene's dialog graph and in fact through the entire story world. This can be both educational and entertaining. Monkey Island is an example of a title that incorporates argumentation tactics in this manner. Popular games like Civilization, Black \& White, and EverQuest, in turn, let the player learn about the world's emergent nature and how they have to live with the worlds they create and the personas they project. In all these games, lines of intellectual inquiry have consequences and discoveries result from explorations.

The same types of explorations can exist in pedagogically oriented dialog graphs. Earlier efforts introduce such possibilities by providing side characters that coach and cajole a player back to the pedagogically preferred path $[11,17]$ of a dialog graph. In the types of storyworlds we currently envision, however, the learning can be just as effective if a sidekick or "window character" regrets aloud the player's decisions and then on its own directly performs the dysfunctional character training and persuading [2]. In theory, the player should even be able to adopt a potential storyworld antagonist's causes and be entertained by helping to support the antagonist's objectives throughout the storyworld, yet suffer no loss of learning as a result. We are finding a lot of success with including such dialog plot regions and archetype roles for cast members to readily fill in, particularly when players grow bored late in a trainingoriented game (see Case Study of this paper).

There have been a number of investigations into conversations with autonomous agents outside of stories and/or in fixed plot graphs and their findings have potential here. Some of the earliest work dealt only with simple animation and kinesthetic issues such as breathing and blinking, lip synching, and facial expressions - things we label as "presentation layer" in Figure 1d [e.g., see 9]. More intriguing, however, is work on the higher layers of Figure $1 \mathrm{~d}$ and how they might be integrated into dialog plots as suggested in Figure 1c. We define the Simulation Layer of an agent as how it performs in the world - e.g., navigation, collision avoidance and damage, and physiologic and health needs. In some characters, we embedded a number of validated reservoir models of body organs and functions [18]. Less realistic models are widely used in popular God games, however, there is never any story designed into it, and players merely ascribe story when uncorrelated events arise. In our work these eventually are intended to provide many potentially engaging training dialog opportunities, particularly with characters that attempt to deny or mislabel their symptoms, risk factors, and lifestyle habits.

Likewise, the Behavior Layer involves characters' emotions and motivations, planning/choosing style, and general personality variables including coping modes. One idea here is to allow NPC or non-player characters' moods and personalities to respond dynamically and emergently to direct player interaction. The NPCs include parameterized models of autonomous, emotive behavior and different types of responses to player actions or dialogs. The Virtual Theatre Project [7] has explored this concept for fixed plot graphs and shown that players perceive significant dramatic variability and storycreating potential, even though the plot is fixed. An early prototype of Heart Sense Game has likewise deployed an autonomous coach/companion that alters its mood, emotion-directed utterances, and physical expressions as a function of where the player strays in the plot or dialog graph and found this reduces player difficulties [17]. In the current research we have eliminated overt coaching, but are attempting to use this idea for key characters so they alter their personality each time you play. This in turn furthers the perceived player variability and sense of 
free play. It also means players must use a different persuasion strategy each time they re-enter a scene.

Silverman, Johns, et al. [18] demonstrate how a number of models from the literature across these two layers (simulation and behavior) can contribute to making agents autonomous and need-reservoir driven in their coping styles and emotive decision making. This is an attempt to move beyond Bates' believable and broad agents [12] into the realm of reliable models of human performance calibrated against field data - an area where learning systems must depart from entertainment.

A final issue facing storytelling in simulated worlds is that it forces the player into what is arguably the worst side of human-computer interaction, that of the computer's poor conversational capabilities. As with voice menu systems on the telephone, the machinegenerated voices are stilted, their ability to handle nuances are poor, and they often misunderstand the speaker. Up to now we use a text to speech system during authoring but replace it with actor voice-overs once the parts are finalized. We completely avoid the speech recognizers and instead rely on dialog menus which raises several difficulties. Specifically, the risk of dialog menus is that the designer has neglected to include options the player would like to see voiced, or if they are voiced, hasn't included mechanics in the other characters to support the idea in the player's head. So far in the case study, however, we have not encountered this difficulty and believe the large degree of free play mentioned in this section tends to minimize the dialog menu risks.

\section{Case Study - Coping With Aesthetic Silos}

Throughout this paper we have been discussing the radically divergent aesthetics that guide different subdisciplines in the edutainment field. It is easiest to explain these in terms of the three dimensions of Figure 2, a graph depicting the tradeoffs that edutainment designers must make between training/content, active gameplay aesthetics, and passive-listening but engaging story and characters. The data points on the graph (A, B, C, D) represent separate edutainment designs that various design groups tried to use AESOP to create and that we further discuss in the remainder of this section. But first we pause to explain the methodology of this case study.

The axes of Figure 2 are a classic example of what is called 'knowledge silos' (or discipline barriers) and the technique of critical systems thinking arose to address such issues and to help with solving problem that require people to reconsider boundaries and potential impacts [4]. Specifically this technique calls for 3 steps: (1) surface stakeholder viewpoints, (2) explore stakeholder issues and tradeoffs, and (3) identify and implement a pluralistic solution. In order to apply this technique, one needs a common design problem, qualified representatives from the respective silo communities, and a willingness of all involved to engage in the 3 steps.

Figure 2 - Three Core Disciplines Strive to Overcome Conflicting Aesthetic Models In the Design Process

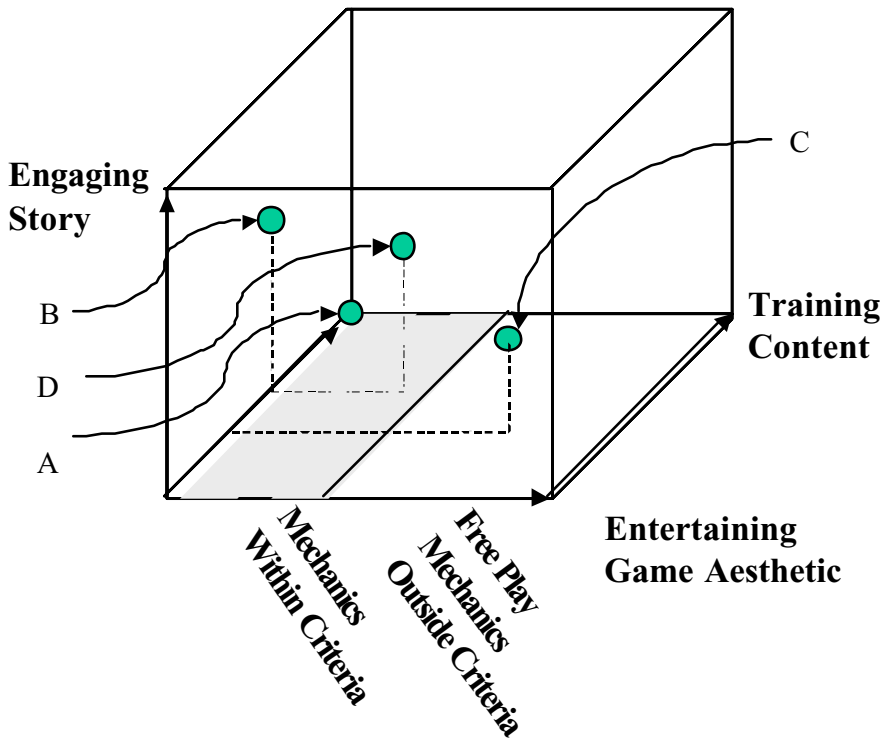

As a common design topic, we were given a grant to author a health behavior intervention in the heart attack domain - what we call Heart Sense Game or HSG. In particular, this is an intervention targeting the delay that pervades heart attack victims deciding to seek treatment. It turns out that people delay for a combination of reasons, some of which are misdiagnosis, but many of which are attitudinal, peer pressure, and other social issues that arise after diagnosis. Since the educational goal of the intervention deals mostly with thinking issues, the design teams were told that the game should probably be based on dialog puzzles, with the player challenged to find a path through various options that persuades a given heart attack victim in the storyworld that 1) he is indeed having a heart attack, and 2) he should go to the hospital quickly. This involved the concept of learning by teaching embedded characters.

The various design teams described in this case study have professional credentials in their respective disciplines. The instructional design and training content team included four mid-career MDs and $\mathrm{PhDs}$ in various health and patient education specialties. The storywriting team included three script writers, two of whom were just starting out with a BS in creative writing, and one of whom has been teaching at area universities for a number of years. The game design team included three selfconfessed game-playing addicts, all of whom had previously created autonomous, embodied agents with game AI, and one of whom had created a 3-D interactive hockey game. At the outset, each of these three teams had 
very little appreciation for (and no skill at) the aesthetics of any other team, and firmly believed their own field included the know-how needed to realize the design. However, they were willing to participate in the effort, and most of them were paid a salary or consultant fee from the grant. We now turn to a discussion of the designs that each team surfaced (step 1 of our methodology) intermingled with some of the critique of those designs.

In traditional computer based training, instructional designers invariably begin with the training objectives [5, 15]. This is what both drives and constrains the design and suggests what lesson modules are needed. Rising to that challenge within the earliest stages of HSG development, the training/content team produced a list of skill and informational deficiencies that the target players were missing. A heart attack victim first goes through a process of symptom denial $(\mathrm{Hx})$ and eventual recognition of the event (Dx). Even after the subject recognizes a serious health threat may exist (Dx), they find other reasons to deny and avoid doing the right thing. There is a large literature on this and we have discussed it at length elsewhere [17]. Persuasion theory (Px) shows that these protests need to be legitimized and the victim needs to be helped to overcome these obstacles often through some form of peeling away of the layers and by adding degrees of confidence. Here the treatment (Tx) is simple, just dial 9-1-1, so the doctors can offset permanent damage to the heart muscle. Persuasion skills are needed to convince victims to overcome these issues.

Dominated by these sobering objectives, the trainers' prototype of the game included three modules [17]: (1) an opening vignette showing an ambulance racing to rescue a victim but arriving too late due to delay; (2) being invited into the ER doctor's office to learn all the training objectives and to be asked to go out into the (virtual) town and help prevent re-occurrences amongst the remaining population, and (3) encounters in the town with characters who must be taken through the entire $\{\mathrm{Hx}|\mathrm{Dx}| \mathrm{Px} \mid \mathrm{Tx}\}$ sequence. This was a thorough design built in Director as a first prototype of HSG.

Building this prototype and putting it in front of focus groups helped to surface a number of fundamental design errors. For one thing, it failed from a game-play perspective since interactivity didn't truly start until the player entered the town, following the 2 minute long (passive viewing) opening scare module and the 5 minute long multi-media lecture in the ER doc's office. By then, any chance of serious involvement of the player in a roleplaying story was basically destroyed. It failed as a drama or interactive theater piece since there was no compelling plot for the user to act as a hero within. Just acting as a Good Samaritan and walking around town diagnosing and persuading folks is not much of an engaging story - and some players balked at entering the storyworld characters' homes. In effect this design is Point A of Figure 2.
The storytellers in turn, took the design challenge and immediately thought about the 3 Ps - plot, people, and place and a hero's journey as in [1]. They came up with a meta-story as follows: Heart Sense Game is a role playing game in which you help the hero try to solve a crime and simultaneously rescue his career and find romance. However, as the hero, some of the many characters you might get clues from, need your help to deal with heart attacks before they or others can help you. Since, for their own reasons, they often don't believe they are having a heart attack or don't want to take care of it promptly, there are significant obstacles to helping these characters to help themselves.

In short, they envisioned a 3-act hero's journey that engages and drives the player and his or her avatar through all the act 2 quests looking for clues to solve the overall crime. From this high level description, they quickly blocked out a 3-act story map with scenes, character archetypes, and backstories. Once the opening act was completed and a given act 2 quest initiated, there would be a character possessing some clues but who had some subset of the $\{\mathrm{Hx}|\mathrm{Dx}| \mathrm{Px} \mid \mathrm{Tx}\}$ sequence. Going through that sequence followed a traditional story arc with tension buildup to climax and resolution. This helped them to define the dialogs for each scene which they authored linearly in the FSM dialog graph editor mentioned earlier.

The problem with the storyteller's design was that the result was linear and largely non-interactive. Writers are not trained in interactive media, and it is difficult for them to envision a human player taking over the role of their central character. While they are skilled at bringing in tension, climax, and drama, they tend to do so by placing the human on 'rails' and in a passive listening role. The resulting design can be thought of as having moved to point B in Figure 2 - far more entertaining, and easing off of trying to teach all the training minutia.

The gamer group of designers approached the design problem as their field recommends by identifying the types of fun they wanted the players to have and the mechanics that would produce that fun $[8,10]$. In addition to some narrative in cut scenes, they envisioned several side games with driving and shooting mechanics. In effect they drove to design point $\mathrm{C}$ of Figure 2. This design makes heavy use of game play, navigation, and twitch reflexes interspersed with much lower doses of the types of stealth learning quests than the story group had programmed into the dialog graphs. However, it ignored the target population of players' demographics and included several mechanics and skills the players would have little interest in or skill at.

In the end, we benefited from all three design perspectives and used them and (in two cases) focus group reactions to eventually help drive the design to point D on Figure 2. For example, using criteria about the 
target population, we eliminated from the gamers' design any form of direct manipulation of objects and the entire exploration mechanic from a terrain perspective (no navigation by under-skilled players). We also eliminated their proposed "magic school bus" tour of the inner workings of veins and clots, as well as any ideas about clot-busting arcade shooting or racing games (age- and gender-inappropriate). The storytellers' version already had incorporated the instructional focus on the $\{\mathrm{Hx}|\mathrm{Dx}| \mathrm{Px} \mid \mathrm{Tx}\}$ sequence. But we had to use the ideas of the gamers, and in particular of earlier Figure 1 to drive the writers to embellish their plot and dialog graphs to include free play in the form of parallel but interwoven stories the player could pursue, and within each story to add alternative persuasion strategies for the dialog menus. In effect we wanted the writers to move from Point B to Point D by adding the following sentence to their story overview: And if you prefer to harm these characters, you are free to do so, but watch out, your own future will be effected as well! In adopting this sentence, the writers' plot graphs eventually began to take on the shape of Figure $1 \mathrm{~b}$ for each scene, rather than being single paths across one set of nodes. When asked, the writers found they could readily think up alternative strategy options for the avatar within each dialog scene. However, they had a lot of trouble figuring out that there might be real consequences for player actions and dialog statements that would cause different branches in the other players, and hence leaps to parallel stories. Here is a retrospective protocol from one of the writers about his scene dialog editing problems: the one thing I was having trouble grasping was exactly how you wanted these three paths to coexist. At first ... I came away with the impression that you wanted the three versions to be more connected, especially after our lengthy discussion about how you wanted the player to be able to move laterally from path to path as a result of his choices. I then wrote a version that was really only one scene, containing three different versions of alternate dialogue, but not diverging at any point. This too was not an accurate representation of what you wanted. Upon more lengthy discussion ... and page upon page of flowcharts and diagrams, I came away with a new understanding of what exactly you were seeking. What I found was that you did not want one scene with some variations in its dialogue, but you also didn't want three distinctly different scenes. What you did want was a combination of the two. A scene that would open and present the player with three different types of choices, but one that would eventually diverge into three different scenes, based on the choices the player made leading up to this diverging point.

The design that represents Point D on Figure 2 was completed, and is currently in production. We placed human voiced-over versions of the first two scenes before a focus group sent over by a local health clinic $(\mathrm{N}=17$.
Avg. Age $=60.5$ ). From 75 to $80 \%$ of the focus group participants found it fun, worth playing again, and worth telling friends about, even though few had ever played videogames before. Furthermore, they understood that this was a game about early reaction to heart attack symptoms. We thus feel there is at least initial evidence that the Critical Systems Methodology was not only internally successful, but also has some external validity as well, though we plan to collect far more data during an upcoming clinical trial of HSG in 2003.

\section{Discussion of Results and Next Steps}

Our research up to this point has revealed two surprising facts. First, there are no environments one can turn to for rapid authoring of pedagogically oriented interactive drama games. While games from other genres are beginning to arrive packaged with sophisticated editing tools, the educational gaming community generally is forced to create non-modifiable games on a per-subject, per-audience basis. There exists a growing number of tried and true guidelines for creating fun games. There exists a huge body of work on the subject of effective methods of education. And narrative has its own effectivity metrics. But, at present, most games are designed from the start with entertainment as the primary goal, with any learning on the part of the player as a beneficial side-effect. Pedagogical games, on the other hand, begin with rigid learning objectives that must be satisfied, which place severe constraints on the design of the game. This tension has created a deep gap between the creators of educational games and the creators of entertainment games, and consequently little mutual benefit is generated from work in either community.

We believe that the solution to this problem lies in the creation of a system that provides the building blocks of interactive storytelling by implementing the inner workings of a variety of gaming devices as composable parts, with their actual arrangement and content determined by the educators. Dialog, character movement, puzzle manipulation, resource models, combat, and other mechanics would be weaved generically into a unified game engine, with the educators able to simply choose which ones suit the story, pedagogical goals of the game, and the needs of the target audience. While a game like HeartSense is inherently dialog-oriented with its focus on persuasion and interpersonal relationships, a game for diabetics may include a resource management component with the player challenged to balance blood sugar through the course of a day. Such a unified engine is becoming an increasingly realistic possibility, with many recent games beginning to blend elements from a variety of others, causing genres, and more importantly game engines, to converge. Given an environment such as this to work 
within, designers can harness the state of the art in the technical aspects of interactive storytelling while staying focused on content creation.

The second surprise is the lack of robust methodologies that help bridge the divergent viewpoints and thinking of those whose disciplines must be united in edutainment titles - training/content experts, storytellers, and game authors. The design case study illustrated how each community is separated by deep silos and uses its prevailing literatures and practices to come up with inadequate designs initially. However, the Critical Systems Thinking seems to be a successful way to cause different design groups to examine the boundaries of their disciplines and to find pluralistic solutions. In helping designers to examine their respective boundaries we found it necessary to actually implement some of the designs and to collect focus group reactions. This helped to sharpen critical awareness and to answer questions about 'design improvement for whom'. Overcoming these mental silos was not an easy task. However, our pluralistic design resultant lead to a prototype that a sample of representative players thought was fun and worth returning to (based on a focus group of 17 players drawn from the target population).

In summary, the entertainment video gaming industry has experienced extraordinary technological growth in the recent past, causing a boom in both in the quality and revenue of these games. Educational games, on the other hand, have lagged behind this trend, as their creation presents major social and pedagogical challenges in addition to technological ones. By providing the technological advances of the entertainment genres in a coherent, accessible format to teams of educators, and developing methodologies for minimizing interdisciplinary friction, we believe that the full potential of educational games can be realized.

\section{ACKNOWLEDGEMENTS}

We gratefully acknowledge the financial support of the National Heart Attack Alert Program of NIH, and the National Library of Medicine PO \# 467-MZ-902060. Also appreciated is the artwork of Joshua Mosley and his many DMD students at Penn, the help of the case design team members (Drs. Holmes, Kimmel, Green, Holmes \& Potok, T.Banden, T.Oksman, K. O'Brien, J. Cornwell), and numerous student helpers and focus group members.

\section{REFERENCES}

[1] Campbell, J., The Hero With a Thousand Faces, Princeton: Bollingen Series/Princeton University Press 1973.

[2] Decker, D., "Anatomy of a Screenplay", First Annual Theory of Story Conference (Storycon), Palm Springs: CA, Sept.2002. www.anatomyofascreenplay.com/book.htm
[3] Filipczak, B., “Training Gets Doomed,” Training, Aug 1997.

[4] Flood, RL, Romm, NR, Critical Systems Thinking: Current Research and Practice, New York: Plenum, 1996.

[5] Gibbons AS, Fairweather, PG, Computer Based Instruction, Englewood Cliffs: Educational Technology Pubs, 1998.

[6] Green, M, Brock, TC. "The Role of Transportation in the Persuasiveness of Public Narratives," J. of Personality and Social Psychology, 79(5), May 2000, pp 701-721.

[7] Hayes-Roth, B, and Rousseau, D., "Improvisational Synthetic Actors with Flexible Personalities." Stanford Knowledge Systems Laboratory Report KSL-97-10, 1997.

[8] Hopson, J., "Behavioral Game Design", Gamasutra, April 27,2001

[9] Lasseter, J., "Principles of Animation Applied to Computer Animation," Computer Graphics, 21(4), July 1987, pp. 35-44.

[10] LeBlanc, M., "Game Design Workshop," Game Developers Conference, San Jose, CA: March 2002.

[11] Marsella, S., Johnson, W.L., and LaBore, C. "Interactive Pedagogical Drama." Proceedings of the $4^{\text {th }}$ International Conference on Autonomous Agents, 2000, pp 301-308.

[12] Mateas, Michael. An Oz-Centric Review of Interactive Drama and Believable Agents. Technical Report CMU-CS-97156, CMU, Pittsburgh, PA. June 1997.

[13] Petty, R. E., \& Cacioppo, J. T.. Communication and persuasion, New York: Springer-Verlag, 1986.

[14] Propp, V, Morphology of the Folktale, Austin: University Texas Press, 1968.

[15] Reigeluth, CM (ed.), Instructional Design Theories and Models, Mahwah: Erlbaum, 1999.

[16] Rickel, J, Gratch, J, Hill, R, et al. "Steve Goes to Bosnia: Towards a New Generation of Virtual Humans for Interactive Experiences". AAAI Spring Symposium on Artificial Intelligence and Interactive Entertainment, Stanford U, CA, March 2001.

[17] Silverman, BG, Holmes, J, Kimmel, S, et al., "Modeling Emotion and Behavior in Animated Personas to Facilitate Human Behavior Change: The Case of the Heart-Sense Game," INFORMS' Jnl of HealthCare Management Science, 4(3), Sept. 2001, 213-228.

[18] Silverman, BG, Johns, M, Weaver, R, et al., "Using Human Behavior Models to Improve the Realism of Synthetic Agents," Cognitive Science Quarterly, 2(3/4), Fall'02, 273-301.

[19] Swartout, W., et al. "Toward the Holodeck: Integrating Graphics, Sound, Character and Story." Proc. Fifth International Conf. Autonomous Agents, May 2001, pp 409-416. 